\title{
HIV awareness and safe sexual behaviors among female sex workers in Kathmandu valley of Nepal
}

This article was published in the following Dove Press journal: HIVIAIDS - Research and Palliative Care

\author{
Sampurna Kakchapati' \\ Nirmal Gautam' \\ Khagendra Prakash $\mathrm{KC}^{2}$ \\ Bir Bahadhur Rawal ${ }^{3}$ \\ 'Department of Mathematics and \\ Computer Science, Faculty of Science \\ and Technology, Prince of Songkla \\ University, Pattani, Thailand; ${ }^{2}$ Health \\ Science Research and Development \\ Concerns, Kathmandu, Nepal; \\ ${ }^{3}$ National Centre for AIDS and STD \\ Control, Kathmandu, Nepal
}

Purpose: Knowledge on HIV and safe sex practices are the main determinants for the prevention and control of HIV/AIDS transmission. The aim of this study was to assess HIV awareness and safe sexual behavior among the female sex workers (FSWs) in the Kathmandu valley of Nepal. Methods: The study was based on data secondary to Integrated Biological and Behavioral Surveillance surveys of 2093 FSWs in Kathmandu valley from 2006 to 2015. Bivariate and multivariate logistic regression models were used to identify the factors associated with knowledge and misconceptions about HIV (BCDEF) and safe sexual practices (consistent condom use with regular clients and nonpaying partners).

Results: FSWs who had secondary education (adjusted odds ratio [AOR] $=2.08,95 \%$ confidence interval $[\mathrm{CI}]=1.60,2.70)$ and visited the drop-in center (DIC) (AOR=1.34, 95\% CI=1.02, 1.75) in the last year had more knowledge and misconceptions about HIV (BCDEF). FSWs who had consistent condom use with nonpaying partners ( $\mathrm{AOR}=1.60,95 \% \mathrm{CI}=1.23,2.09$ ), had a HIV test $(\mathrm{AOR}=1.34,95 \% \mathrm{CI}=1.02,1.76)$, met peer educators $(\mathrm{PEs})(\mathrm{AOR}=1.49,95 \% \mathrm{CI}=1.17,1.91)$ and visited the DIC in the last year $(\mathrm{AOR}=1.32,95 \% \mathrm{CI}=1.01,1.72)$ had a higher chance of condom use with clients. Married FSWs $(\mathrm{AOR}=2.23,95 \% \mathrm{CI}=1.57,3.17)$ and FSWs who met PEs in the last year were more likely to have condom use with nonpaying partners $(\mathrm{AOR}=1.42$, $95 \% \mathrm{CI}=1.04,1.93)$.

Conclusion: Knowledge and misconceptions about HIV has decreased over the years among FSWs; however, consistent condom use with regular clients and nonpaying partners has significantly increased. HIV intervention programs were strongly associated with safe sexual practices among FSWs and need to be strengthened.

Keywords: HIV knowledge, misconceptions, sex work, safe sex

\section{Introduction}

Female sex workers (FSWs) are women who have been professionally involved in sex for money or any gifts for their source of income. ${ }^{1}$ Due to their involvement, FSWs are considered highly responsible for transmitting HIV/AIDS and sexually transmitted infections (STIs) from high-risk to low-risk population. ${ }^{2}$ Globally, the prevalence of FSWs remains high and varies by geographical regions. It was found that the estimated prevalence of HIV infection among FSWs was $0.3 \%$ in the Middle East and North Africa and $29.3 \%$ in the sub-Saharan region, while in the developed countries it was about $1.8 \%$ of the total female population. ${ }^{3}$

In Nepal, the prevalence of HIV/AIDS is high in key population, namely FSWs, people who inject drugs, men having sex with men and migrants and their spouse. ${ }^{4,5}$ Nonetheless, it was found that the number of FSWs is increasing day
Correspondence: Sampurna Kakchapati Department of Mathematics and Computer Science, Faculty of Science and Technology, Prince of Songkla University, Rusamalie-4, Pattani 94000, Thailand $\mathrm{Tel}+66733 \mathrm{I} 3928$ (Extension 50) Fax +6673372179 Email sampurna.k@psu.ac.th 
by day with estimates of 43,829 and 54,197 in 2012 and 2015, respectively. ${ }^{6}$ Among the different regions studied, Kathmandu valley had the highest number of FSWs, which was about 9789-11,670. ${ }^{7}$ This growing number of FSWs in Kathmandu valley is because of sociocultural and economic factors, instability of the political situation and gender disparities, which lead to poverty, domestic violence and other family-related psychological stresses, compelling the affected women to enter into the sex trade. ${ }^{7,8}$

The poor knowledge on reproductive health often does not allow FSWs to maintain their sexual health needs. Hence, negotiation regarding safe sexual behavior has been a cause of violence, rape and other types of physical abuse by the male partners. ${ }^{8,9}$ Additionally, it was also found that due to the patriarchal society, females have restricted access to literacy, job opportunities and their fundamental rights. ${ }^{10}$ These factors make females dependent, and so they are exposed to abuse. ${ }^{11}$ Therefore, the prevalence of HIV has not improved significantly, and has become an epidemic, with approximately 39,249 people infected in 2014; although earlier it decreased from 50,000 to 40,723 in 2012 and 2013, respectively. ${ }^{12,13}$ These alarming trends for HIV/AIDS and STIs are further related to the fact that most of the infected people are unaware of their status; hence, HIV/AIDS and STIs are still common causes of death among women of reproductive age groups in Nepal. ${ }^{13}$ The available evidence suggests that the HIV/AIDS epidemic among FSWs in Nepal remained stagnant from 2004 to 2016, suggesting the need to reduce the prevalence of HIV among FSWs. The prevalence of syphilis dropped from 2002 to 2012 with significant increases in 2015 and 2016. The recent increase in syphilis has become a major concern and needs to be further investigated. ${ }^{14}$ In Nepal, education on comprehensive knowledge and misconceptions about HIV and HIV prevention programs (outreach educators [OEs]/peer educators [PEs]/community mobilizers [CMs], drop-in center [DIC], STI clinic and HIV testing and counseling (HTC) center) is regularly provided to FSWs. However, recent studies have also revealed that unsafe sexual habits and low access to information on HIV and HIV prevention programs among FSWs are the main risks of having HIV/AIDS and STIs. ${ }^{14-16}$ Furthermore, inconsistent condom use with clients and with nonpaying partners over the past 12 months was well documented in other studies. ${ }^{14,17-19}$ However, there is hardly any information regarding HIV/AIDS among FSWs. The aim of this study was to investigate the knowledge on HIV/AIDS and safe sex practices among FSWs and to assess the risk factors associated with HIV/AIDS in the Kathmandu valley of Nepal using a large data set from biobehavioral surveys.

\section{Methods}

This study used data from cross-sectional biobehavioral surveys in 2006, 2008, 2011 and 2015 which aimed to sample 2093 FSWs using two-stage cluster sampling in Kathmandu valley. Eligible FSWs were defined as women aged 16 years and above who reported being paid in cash or kind for sex with a male within the last 6 months.

Face-to-face interviews were conducted by trained field workers in the local language, Nepali, using a structured questionnaire that included questions on background characteristics (age, year, region, education, marital status, type of sex work), sexual behaviors (duration of their involvement in the sex trade, average number of clients, total number of working days), injecting behaviors (use of drugs, injecting practices) and comprehensive knowledge on HIV (ABC) and reflected a deeper understanding of HIV. Similarly, knowledge and misconceptions about HIV (BCDEF) indicated the false understanding of HIV and exposure to the HIV prevention program (HIV test, met with OEs/PEs/CMs, visited DIC, visited STI clinic and visited HTC center).

Knowledge on $\mathrm{ABC}$ was defined as being aware of $\mathrm{A}$ (abstinence from sex), B (monogamy or being faithful to one partner or avoiding multiple sex partners) and $\mathrm{C}$ (consistent and correct condom use or use of a condom during every sex act) as HIV-preventive measures. Additionally, DEF referred to knowledge that a healthy-looking person can be infected with HIV (D), a person cannot get HIV from a mosquito bite (E) and one cannot get HIV by sharing a meal with an HIV-infected person (F).

The sex partners of the FSWs were categorized as clients, regular clients, nonpaying partners and other partners. Clients and regular partners were those partners who pay for sexual contact. Nonpaying partners included boyfriends, husbands, intimate partners or those who do not pay for sexual services. Partners other than clients, husbands and the male friend(s) were categorized as other partners which included massage parlor owners and police officials. Other partners mostly do not pay for sexual contact with FSWs.

Ethical clearances and verbal informed consent approval were obtained from the Nepal Health Research Council. Verbal informed and witnessed consent was obtained from all the FSWs prior to the interview. As the age of majority in Nepal is 16, no parental consent was required.

Logistic regression analysis was performed to examine the effects of determinants of HIV knowledge and consistent 
condom use with regular clients and nonpaying partners. Initially, explanatory determinants were included in the model one at a time to examine their univariate relationship with the outcome. Multivariate logistic regressions were used to identify the most important determinants of each outcome. A $P$-value of $<0.05$ was used to define statistical significance. Adjusted odds ratios (AORs) as well as their 95\% confidence intervals ( $95 \% \mathrm{CIs}$ ) were used to depict the independent relationship between predictors and dependent variables. The $\mathrm{R}$ program was used for statistical analysis.

\section{Results}

About 2093 FSWs were recruited in Kathmandu valley during the period 2006-2015. Table 1 shows the background characteristics, sexual behaviors, injecting behaviors and participation in HIV prevention programs among FSWs. A majority of FSWs worked on the streets. Most of the FSWs were above 20 years, had primary and secondary education and were married. The duration of sex work of more than half of the FSWs interviewed was more than 1 year. A majority of FSWs had more than one sexual partner per day and worked less than 2 days per week. About $70 \%$ of FSWs had consistent condom use with regular clients; however, one-fifth of the FSWs had consistent condom use with nonpaying partners. A majority of FSWs reported alcohol consumption, but the drug use was low (7\%). About $29 \%$ of F SWs had knowledge and misconceptions about HIV. Most of the FSWs met OEs/ $\mathrm{PEs} / \mathrm{CMs}$ in the last year. More than one-third of the FSWs had visited DIC and HTC center in the last year, whereas less than one-third of the FSWs visited STI clinic in the last year.

Table 2 shows the factors associated with knowledge and misconceptions about HIV (BCDEF) among FSWs in Kathmandu valley for each determinant. The results on year, education and DIC visits in the last year were significantly associated with knowledge on BCDEF in multivariate analysis $(P<0.05)$. Knowledge on BCDEF was found to be significantly decreased in 2015 compared to the 2008 survey. FSWs who had secondary education were more likely to have knowledge and misconceptions about $\mathrm{HIV}(\mathrm{AOR}=2.08,95 \% \mathrm{CI}=1.60,2.70)$ compared to those who had primary education $(\mathrm{AOR}=1.61,95 \% \mathrm{CI}=1.25$, 2.08). Furthermore, FSWs who visited the DIC in the last year had more knowledge on BCDEF (AOR $=1.34,95 \% \mathrm{CI}=1.02$, $1.75)$ than those who did not visit DIC in the last year.

Table 3 shows the association between determinants and consistent condom use with regular clients. The proportion of FSWs who had consistent condom use with regular clients was found to be $71 \%$. In multivariate analysis, consistent use of condoms with nonpaying partners, no exposure to the HIV test, meeting OEs/PEs/CMs in the last year, visiting DIC
Table I Background characteristics, sexual behaviors, injecting behaviors and HIV prevention programs among FSWs (N=2093)

\begin{tabular}{|c|c|c|}
\hline Characteristics & $\begin{array}{l}\text { Number } \\
(\mathrm{N}=\mathbf{2 0 9 3})\end{array}$ & Percentage \\
\hline \multicolumn{3}{|l|}{ Year } \\
\hline 2006 & 500 & 23.9 \\
\hline 2008 & 500 & 23.9 \\
\hline 2011 & 593 & 28.3 \\
\hline 2015 & 500 & 23.9 \\
\hline \multicolumn{3}{|l|}{ Type } \\
\hline Establishment & 832 & 39.8 \\
\hline Street & $|26|$ & 60.2 \\
\hline \multicolumn{3}{|l|}{ Age (years) } \\
\hline Below 20 & 678 & 32.4 \\
\hline $21-29$ & 840 & 40.1 \\
\hline 30 and above & 575 & 27.5 \\
\hline \multicolumn{3}{|l|}{ Education } \\
\hline None & 660 & 31.5 \\
\hline Primary & 771 & 36.8 \\
\hline Secondary and above & 662 & 31.6 \\
\hline \multicolumn{3}{|l|}{ Marital status } \\
\hline Single & 481 & 23 \\
\hline Married & 1059 & 50.6 \\
\hline Separated & 553 & 26.4 \\
\hline \multicolumn{3}{|l|}{ Duration of sex work } \\
\hline Less than I year & 905 & 43.2 \\
\hline $\mathrm{I}-3$ years & 730 & 34.9 \\
\hline More than 3 years & 458 & 21.9 \\
\hline \multicolumn{3}{|c|}{$\begin{array}{l}\text { Total number of sexual partners in } \\
\text { a week }\end{array}$} \\
\hline One and less than one & 98 & 4.7 \\
\hline More than one & 1995 & 95.3 \\
\hline \multicolumn{3}{|c|}{ Number of working days in a week } \\
\hline Two and less than two & 1989 & 95 \\
\hline More than two & 104 & 5.0 \\
\hline \multicolumn{3}{|c|}{ Consistent condom use with } \\
\hline \multicolumn{3}{|c|}{ regular clients } \\
\hline No & 610 & 29.1 \\
\hline Yes & 1483 & 70.9 \\
\hline \multicolumn{3}{|c|}{ Consistent condom use with } \\
\hline \multicolumn{3}{|c|}{ nonpaying partners } \\
\hline No & 1642 & 78.5 \\
\hline Yes & 451 & 21.5 \\
\hline \multicolumn{3}{|l|}{ Ever used drugs } \\
\hline No & 1948 & 93.1 \\
\hline Yes & 145 & 6.9 \\
\hline \multicolumn{3}{|l|}{ Alcohol consumption } \\
\hline No & 585 & 28 \\
\hline Yes & 1508 & 72 \\
\hline \multicolumn{3}{|l|}{ Ever had an HIV test } \\
\hline No & 970 & 46.3 \\
\hline Yes & 1123 & 53.7 \\
\hline \multicolumn{3}{|c|}{$\begin{array}{l}\text { Knowledge and misconceptions } \\
\text { (BCDEF) }\end{array}$} \\
\hline No & 1494 & 71.4 \\
\hline Yes & 599 & 28.6 \\
\hline \multicolumn{3}{|c|}{ Met OEs/PEs/CMs in the last year } \\
\hline No & 528 & 25.2 \\
\hline Yes & 1565 & 74.8 \\
\hline
\end{tabular}

(Continued) 
Table I (Continued)

\begin{tabular}{lll}
\hline Characteristics & $\begin{array}{l}\text { Number } \\
(\mathbf{N}=2093)\end{array}$ & Percentage \\
\hline Visited DIC in the last year & 1356 & 64.8 \\
No & 737 & 35.2 \\
Yes & & \\
Visited STI clinic in the last year & 1420 & 67.8 \\
No & 673 & 32.2 \\
Yes & & \\
Visited HTC center in the last year & 1349 & 64.5 \\
No & 744 & 35.5 \\
Yes &
\end{tabular}

Abbreviations: BCDEF, B (monogamy or being faithful to one partner or avoiding multiple sex partners), $C$ (consistent and correct condom use or use of a condom during every sex act), D (healthy-looking person can be infected with HIV), E (a person cannot get HIV from a mosquito bite), and $F$ (one cannot get HIV by sharing a meal with an HIV-infected person); CMs, community mobilizers; DIC, drop-in center; FSWs, female sex workers; HTC, HIV testing and counseling; OEs, outreach educators; PEs, peer educators; STI, sexually transmitted infection. in the last year and visiting STI clinic in the last year were significantly associated with consistent condom use with regular clients $(P<0.05)$. There was no statistically significant association of knowledge and misconceptions about HIV with consistent condom use with regular clients. FSWs who had consistent condom use with nonpaying partners had a higher rate of condom use with clients $(\mathrm{AOR}=1.60,95 \% \mathrm{CI}=1.23$, 2.09). Moreover, FSWs who did the HIV test had a higher rate of condom use with clients ( $\mathrm{AOR}=1.34,95 \% \mathrm{CI}=1.02,1.76)$ than those who did not use condoms. Lastly, FSWs who met $\mathrm{OEs} / \mathrm{PEs} / \mathrm{CMs}(\mathrm{AOR}=1.49,95 \% \mathrm{CI}=1.17,1.91)$ and visited $\mathrm{DIC}$ in the last year $(\mathrm{AOR}=1.32,95 \% \mathrm{CI}=1.01,1.72)$ had a higher rate of condom use with clients.

Table 4 shows the association between determinants and consistent condom use with nonpaying regular partners. In

Table 2 Factors associated with knowledge and misconceptions about HIV (BCDEF) among FSWs in Kathmandu valley

\begin{tabular}{|c|c|c|c|c|c|c|}
\hline \multirow[t]{2}{*}{ Variable } & \multicolumn{2}{|c|}{ Knowledge on BCDEF } & \multirow{2}{*}{$\begin{array}{l}\text { Univariate OR } \\
(95 \% \mathrm{Cl})\end{array}$} & \multirow[t]{2}{*}{$P$-value } & \multirow{2}{*}{$\begin{array}{l}\text { Multiple OR } \\
(95 \% \mathrm{Cl})\end{array}$} & \multirow[t]{2}{*}{$P$-value } \\
\hline & $\begin{array}{l}\text { Yes (N=599, } \\
29 \%), \text { n (\%) }\end{array}$ & $\begin{array}{l}\text { No }(N=1494, \\
71 \%), \text { n (\%) }\end{array}$ & & & & \\
\hline \multicolumn{7}{|l|}{ Year } \\
\hline 2006 & $15 \mid(30.2)$ & $349(69.8)$ & I & & I & \\
\hline 2008 & $182(36.4)$ & $318(63.6)$ & $1.32(1.02,1.72)$ & 0.04 & $1.37(1.03,1.83)$ & 0.028 \\
\hline 2011 & $180(30.4)$ & $413(69.6)$ & $\mathrm{I} .0 \mathrm{I}(0.78, \mathrm{I} .3 \mathrm{I})$ & 0.865 & $0.99(0.74, \mathrm{I} .3 \mathrm{I})$ & 0.966 \\
\hline 2015 & $86(17.2)$ & $4 \mid 4(82.8)$ & $0.48(0.36,0.65)$ & $<0.001$ & $0.53(0.37,0.75)$ & 0.001 \\
\hline Type & & & & 0.152 & & \\
\hline Establishment & $224(26.9)$ & $608(73.1)$ & 1 & & & \\
\hline Street & $375(29.7)$ & $886(70.3)$ & $1.15(0.95,1.4)$ & & & \\
\hline \multicolumn{7}{|l|}{ Age (years) } \\
\hline Below 20 & $336(49.6)$ & $342(50.4)$ & 1 & & & \\
\hline $21-29$ & 394 (46.9) & $446(53.1)$ & $1.17(0.94,1.47)$ & 0.098 & & \\
\hline 30 and above & $273(47.5)$ & $302(52.5)$ & I $(0.78,1.28)$ & 0.110 & & \\
\hline \multicolumn{7}{|l|}{ Education } \\
\hline None & $147(22.3)$ & $513(77.7)$ & 1 & & I & \\
\hline Primary & $229(29.7)$ & $542(70.3)$ & $1.47(1.16,1.87)$ & $<0.001$ & $1.61(1.25,2.08)$ & $<0.001$ \\
\hline Secondary and above & $223(33.7)$ & $439(66.3)$ & $1.77(1.39,2.26)$ & $<0.001$ & $2.08(1.60,270)$ & $<0.001$ \\
\hline \multicolumn{7}{|l|}{ Marital status } \\
\hline Single & $129(26.8)$ & $352(73.2)$ & 1 & & & \\
\hline Married & $308(29.1)$ & $75 I$ (70.9) & $1.12(0.88,1.42)$ & 0.244 & & \\
\hline Separated & $162(29.3)$ & $391(70.7)$ & $1.13(0.86,1.48)$ & 0.576 & & \\
\hline \multicolumn{7}{|l|}{ Duration of sex work } \\
\hline Less than I year & $243(26.9)$ & $662(73.1)$ & & & & \\
\hline I-3 years & $221(30.3)$ & $509(69.7)$ & $1.18(0.95,1.47)$ & 0.137 & & \\
\hline More than 3 years & $135(29.5)$ & $323(70.5)$ & $1.14(0.89,1.46)$ & 0.305 & & \\
\hline Total number of sexual partners in a week & & & & 0.753 & & \\
\hline One and less than one & $24(24.5)$ & $74(75.5)$ & 1 & & & \\
\hline More than one & $575(28.8)$ & $1420(7 \mid .2)$ & $1.25(0.78,2)$ & & & \\
\hline Number of working days in a week & & & & 0.983 & & \\
\hline Two and less than two & $576(29)$ & $14 \mid 3(7 \mid)$ & I & & & \\
\hline More than two & $23(22.1)$ & 81 (77.9) & $0.69(0.43,1.1)$ & & & \\
\hline Consistent condom use with regular clients & & & & 0.993 & & \\
\hline No & $173(28.4)$ & $437(71.6)$ & I & & & \\
\hline Yes & $426(28.7)$ & $1057(71.3)$ & $1.01(0.82,1.25)$ & & & \\
\hline
\end{tabular}


Table 2 (Continued)

\begin{tabular}{|c|c|c|c|c|c|c|}
\hline \multirow[t]{2}{*}{ Variable } & \multicolumn{2}{|c|}{ Knowledge on BCDEF } & \multirow{2}{*}{$\begin{array}{l}\text { Univariate OR } \\
(95 \% \mathrm{CI})\end{array}$} & \multirow[t]{2}{*}{$P$-value } & \multirow{2}{*}{$\begin{array}{l}\text { Multiple OR } \\
(95 \% \mathrm{Cl})\end{array}$} & \multirow[t]{2}{*}{$P$-value } \\
\hline & $\begin{array}{l}\text { Yes } \\
(N=599, \\
29 \%), n(\%)\end{array}$ & $\begin{array}{l}\text { No } \\
(N=1494, \\
71 \%), n(\%)\end{array}$ & & & & \\
\hline Consistent condom use with nonpaying & & & & 0.01 & & 0.42 \\
\hline \multicolumn{7}{|l|}{ partners } \\
\hline No & $490(29.8)$ & $1152(70.2)$ & 1 & & 1 & \\
\hline Yes & $109(24.2)$ & $342(75.8)$ & $0.75(0.59,0.95)$ & & $0.89(0.68,1.16)$ & \\
\hline Knowledge on ABC & & & & $<0.001$ & & $<0.001$ \\
\hline No & $179(16.4)$ & $911(83.6)$ & 1 & & 1 & \\
\hline Yes & 420 (41.9) & $583(58.1)$ & $3.67(2.99,4.49)$ & & $3.36(2.72,4.15)$ & \\
\hline Ever used drugs & & & & 0.725 & & \\
\hline No & $556(28.5)$ & I $392(7 \mid .5)$ & 1 & & & \\
\hline Yes & $43(29.7)$ & $102(70.3)$ & $1.06(0.73,1.53)$ & & & \\
\hline Alcohol consumption & & & & 0.111 & & \\
\hline No & $174(29.7)$ & $4 I I(70.3)$ & 1 & & & \\
\hline Yes & $425(28.2)$ & $1083(71.8)$ & $0.93(0.75,1.14)$ & & & \\
\hline Ever had an HIV test & & & & 0.001 & & 0.08 \\
\hline No & $245(25.3)$ & $725(74.7)$ & 1 & & I & \\
\hline Yes & $354(31.5)$ & $769(68.5)$ & $1.36(1.12,1.65)$ & & $1.29(0.96,1.73)$ & \\
\hline Met OEs/PEs/CMs in the last year & & & & 0.007 & & 0.38 \\
\hline No & $127(24.1)$ & $401(75.9)$ & 1 & & 1 & \\
\hline Yes & $472(30.2)$ & $1093(69.8)$ & $1.36(1.09,1.7 I)$ & & $1.12(0.85,1.48)$ & \\
\hline Visited DIC in the last year & & & & 0.01 & & 0.03 \\
\hline No & $364(26.8)$ & $992(73.2)$ & 1 & & 1 & \\
\hline Yes & $235(31.9)$ & $502(68.1)$ & $1.28(1.05,1.55)$ & & $1.34(1.02,1.75)$ & \\
\hline Visited STI clinic in the last year & & & & 0.0008 & & 0.63 \\
\hline No & $374(26.3)$ & $1046(73.7)$ & I & & 1 & \\
\hline Yes & $225(33.4)$ & $448(66.6)$ & I.4 (I.I5, I.7I) & & $0.93(0.7 \mathrm{I}, \mathrm{I} .22)$ & \\
\hline Visited HTC center in the last year & & & & $<0.001$ & & 0.50 \\
\hline No & $340(25.2)$ & $1009(74.8)$ & I & & 1 & \\
\hline Yes & $259(34.8)$ & $485(65.2)$ & $1.58(1.3,1.93)$ & & $\mathrm{I} .1 \mathrm{I}(0.8 \mathrm{I}, \mathrm{I} .5 \mathrm{I})$ & \\
\hline
\end{tabular}

Table 3 Factors associated with consistent condom use with regular clients among FSWs in Kathmandu valley

\begin{tabular}{|c|c|c|c|c|c|c|}
\hline \multirow[t]{2}{*}{ Variable } & \multicolumn{2}{|c|}{$\begin{array}{l}\text { Consistent condom use } \\
\text { with regular clients }\end{array}$} & \multirow[t]{2}{*}{$\begin{array}{l}\text { Univariate OR } \\
(95 \% \mathrm{Cl})\end{array}$} & \multirow[t]{2}{*}{$P$-value } & \multirow[t]{2}{*}{$\begin{array}{l}\text { Multiple OR } \\
(95 \% \mathrm{CI})\end{array}$} & \multirow[t]{2}{*}{$P$-value } \\
\hline & $\begin{array}{l}\text { Yes (N=I483, } \\
71 \%), n(\%)\end{array}$ & $\begin{array}{l}\text { No }(N=6 I 0, \\
29 \%), \text { n (\%) }\end{array}$ & & & & \\
\hline \multicolumn{7}{|l|}{ Year } \\
\hline 2006 & $340(68)$ & $160(32)$ & I & & I & \\
\hline 2008 & $320(64)$ & $180(36)$ & $0.84(0.64,1.09)$ & 0.910 & $0.97(0.73,1.27)$ & 0.832 \\
\hline 2011 & 437 (73.7) & $156(26.3)$ & $1.32(\mathrm{I} .0 \mathrm{I}, \mathrm{I} .7 \mathrm{I})$ & 0.043 & $1.24(0.94,1.63)$ & 0.120 \\
\hline 2015 & $386(77.2)$ & $114(22.8)$ & $1.59(1.2,2.11)$ & 0.023 & $1.28(0.94,1.75)$ & 0.108 \\
\hline Type & & & & 0.868 & & \\
\hline Establishment & $586(70.4)$ & $246(29.6)$ & I & & & \\
\hline Street & 897 (7I.I) & $364(28.9)$ & $1.03(0.85,1.25)$ & & & \\
\hline \multicolumn{7}{|l|}{ Age (years) } \\
\hline Below 20 & $475(70.1)$ & 203 (29.9) & 1 & & & \\
\hline $21-29$ & 602 (7I.7) & $238(28.3)$ & I.08 $(0.87,1.35)$ & 0.792 & & \\
\hline 30 and above & $406(70.6)$ & $169(29.4)$ & $\mathrm{I} .03(0.8, \mathrm{I} .3 \mathrm{I})$ & 0.234 & & \\
\hline
\end{tabular}


Table 3 (Continued)

\begin{tabular}{|c|c|c|c|c|c|c|}
\hline \multirow[t]{2}{*}{ Variable } & \multicolumn{2}{|c|}{$\begin{array}{l}\text { Consistent condom use } \\
\text { with regular clients }\end{array}$} & \multirow[t]{2}{*}{$\begin{array}{l}\text { Univariate OR } \\
(95 \% \mathrm{Cl})\end{array}$} & \multirow[t]{2}{*}{$P$-value } & \multirow[t]{2}{*}{$\begin{array}{l}\text { Multiple OR } \\
(95 \% \mathrm{CI})\end{array}$} & \multirow[t]{2}{*}{$P$-value } \\
\hline & $\begin{array}{l}\text { Yes (N=1483, } \\
71 \%), n(\%)\end{array}$ & $\begin{array}{l}\text { No }(N=610 \\
29 \%), n(\%)\end{array}$ & & & & \\
\hline \multicolumn{7}{|l|}{ Education } \\
\hline None & $452(68.5)$ & $208(3 \mid .5)$ & I & & I & \\
\hline Primary & $537(69.6)$ & $234(30.4)$ & $1.06(0.84,1.32)$ & 0.826 & $\mathrm{I} .04(0.83,0.3 \mathrm{I})$ & 0.692 \\
\hline Secondary and above & $494(74.6)$ & $168(25.4)$ & $1.35(1.06,1.72)$ & 0.031 & $1.34(1.05,1.72)$ & 0.018 \\
\hline \multicolumn{7}{|l|}{ Marital status } \\
\hline Single & $338(70.3)$ & I 43 (29.7) & I & & & \\
\hline Married & $740(69.9)$ & $319(30.1)$ & $0.98(0.78,1.24)$ & 0.203 & & \\
\hline Separated & $405(73.2)$ & $148(26.8)$ & $1.16(0.88,1.52)$ & 0.458 & & \\
\hline \multicolumn{7}{|l|}{ Duration of sex work } \\
\hline Less than I year & $618(68.3)$ & $287(3 \mid .7)$ & & & & \\
\hline $1-3$ years & $532(72.9)$ & $198(27.1)$ & $1.25(1.01,1.55)$ & 0.176 & & \\
\hline More than 3 years & $333(72.7)$ & $125(27.3)$ & $1.24(0.96,1.59)$ & 0.716 & & \\
\hline Total number of sexual partners & & & & 0.445 & & \\
\hline One and less than one & $70(7 \mid .4)$ & $28(28.6)$ & 1 & & & \\
\hline More than one & $1413(70.8)$ & $582(29.2)$ & $0.97(0.62,1.52)$ & & & \\
\hline Number of working days in a week & & & & 0.218 & & \\
\hline Two and less than two & $1403(70.5)$ & $586(29.5)$ & 1 & & & \\
\hline More than two & $80(76.9)$ & $24(23.1)$ & $1.39(0.87,2.22)$ & & & \\
\hline $\begin{array}{l}\text { Consistent condom use with nonpaying } \\
\text { partners }\end{array}$ & & & & $<0.001$ & & 0.0004 \\
\hline No & II $23(68.4)$ & $519(31.6)$ & I & & I & \\
\hline Yes & $360(79.8)$ & $91(20.2)$ & $1.83(1.42,2.35)$ & & $1.60(1.23,2.09)$ & \\
\hline Knowledge on ABC & & & & 0.404 & & \\
\hline No & $772(70.8)$ & $318(29.2)$ & I & & & \\
\hline Yes & $711(70.9)$ & $292(29.1)$ & $\mathrm{I}(0.83, \mid .2 \mathrm{I})$ & & & \\
\hline Knowledge on BCDEF & & & & 0.872 & & \\
\hline No & $1057(70.7)$ & $437(29.3)$ & 1 & & & \\
\hline Yes & $426(7 I .1)$ & $173(28.9)$ & $1.02(0.83,1.25)$ & & & \\
\hline Ever used drugs & & & & 0.154 & & \\
\hline No & I385 (7I.I) & $563(28.9)$ & I & & & \\
\hline Yes & $98(67.6)$ & $47(32.4)$ & $0.85(0.59,1.22)$ & & & \\
\hline Alcohol consumption & & & & 0.135 & & \\
\hline No & $403(68.9)$ & $182(3||)$. & 1 & & & \\
\hline Yes & $1080(71.6)$ & $428(28.4)$ & I.3I $(0.93,1.83)$ & & & \\
\hline Ever had an HIV test & & & & $<0.001$ & & 0.035 \\
\hline No & $628(64.7)$ & $342(35.3)$ & $\mathrm{l}$ & & I & \\
\hline Yes & $855(76.1)$ & $268(23.9)$ & $1.74(1.44,2.1)$ & & $1.34(1.02,1.76)$ & \\
\hline Met OEs/PEs/CMs in the last year & & & & $<0.001$ & & 0.001 \\
\hline No & $321(60.8)$ & $207(39.2)$ & 1 & & & \\
\hline Yes & II $62(74.2)$ & $403(25.8)$ & $1.86(1.51,2.29)$ & & $1.49(1.17,1.91)$ & \\
\hline Visited DIC in the last year & & & & $<0.001$ & & 0.035 \\
\hline No & $905(66.7)$ & $45 \mathrm{I}(33.3)$ & I & & I & \\
\hline Yes & $578(78.4)$ & $159(21.6)$ & $\mathrm{I} .8 \mathrm{I}(\mathrm{I} .47,2.23)$ & & $1.32(1.01,1.72)$ & \\
\hline Visited STI clinic in the last year & & & & $<0.001$ & & 0.416 \\
\hline No & $982(69.2)$ & $438(30.8)$ & 1 & & I & \\
\hline Yes & $50 \mathrm{I}(74.4)$ & $172(25.6)$ & I.3 (1.06, I.6) & & $0.89(0.68,1.16)$ & \\
\hline Visited HTC center in the last year & & & & 0.001 & & 0.882 \\
\hline No & $920(68.2)$ & $429(31.8)$ & 1 & & I & \\
\hline Yes & $563(75.7)$ & $181(24.3)$ & $1.45(1.18,1.78)$ & & $0.97(0.7 \mathrm{I}, \mathrm{I} .32)$ & \\
\hline
\end{tabular}

Note: Statistically significant values are shown in bold.

Abbreviations: ABC, A (abstinence from sex), B (monogamy or being faithful to one partner or avoiding multiple sex partners), and C (consistent and correct condom use or use of a condom during every sex act); BCDEF, B (monogamy or being faithful to one partner or avoiding multiple sex partners), $C$ (consistent and correct condom use or use of a condom during every sex act), D (healthy-looking person can be infected with HIV), $E$ (a person cannot get HIV from a mosquito bite), and $F$ (one cannot get HIV by sharing a meal with an HIV-infected person); Cl, confidence interval; CMs, community mobilizers; DIC, drop-in center; FSWs, female sex workers; HTC, HIV testing and counseling; OEs, outreach educators; OR, odds ratio; PEs, peer educators; STI, sexually transmitted infection. 
Table 4 Factors associated with consistent condom use with nonpaying regular partners among FSWs in Kathmandu valley

\begin{tabular}{|c|c|c|c|c|c|c|}
\hline \multirow[t]{2}{*}{ Variable } & \multicolumn{2}{|c|}{$\begin{array}{l}\text { Consistent condom use with } \\
\text { nonpaying partners }\end{array}$} & \multirow[t]{2}{*}{$\begin{array}{l}\text { Univariate OR } \\
(95 \% \mathrm{Cl})\end{array}$} & \multirow[t]{2}{*}{$P$-value } & \multirow[t]{2}{*}{$\begin{array}{l}\text { Multiple OR } \\
(95 \% \mathrm{Cl})\end{array}$} & \multirow[t]{2}{*}{$P$-value } \\
\hline & $\begin{array}{l}\text { Yes }(N=45 \mathrm{I} \\
2 \mathrm{I} \%), \mathrm{n}(\%)\end{array}$ & $\begin{array}{l}\text { No }(N=1642 \text {, } \\
79 \%), \text { n (\%) }\end{array}$ & & & & \\
\hline \multicolumn{7}{|l|}{ Year } \\
\hline 2006 & $90(18)$ & $410(82)$ & I & & 1 & \\
\hline 2008 & $56(11.2)$ & $444(88.8)$ & $0.57(0.4,0.82)$ & 0.030 & $0.63(0.43,0.9 \mathrm{I})$ & 0.023 \\
\hline 2011 & 94 (I5.9) & $499(84.1)$ & $0.86(0.62,1.18)$ & 0.164 & $0.74(0.53,1.03)$ & 0.147 \\
\hline 2015 & $211(42.2)$ & $289(57.8)$ & $3.33(2.49,4.44)$ & $<0.001$ & $2.89(2.08,4.01)$ & $<0.001$ \\
\hline Type & & & & 0.642 & & \\
\hline Establishment & $173(20.8)$ & $659(79.2)$ & I & & & \\
\hline Street & $278(22)$ & $983(78)$ & $\mathrm{I} .08(0.87, \mathrm{I} .33)$ & & & \\
\hline \multicolumn{7}{|l|}{ Age (years) } \\
\hline Below 20 & $118(17.4)$ & $560(82.6)$ & I & & 1 & \\
\hline $21-29$ & $184(21.9)$ & $656(78.1)$ & $1.33(1.02,1.72)$ & 0.02 & $0.97(0.7 \mathrm{I}, \mathrm{I} .33)$ & 0.974 \\
\hline 30 and above & $149(25.9)$ & $426(74.1)$ & $1.66(1.26,2.18)$ & $<0.001$ & $0.94(0.64,1.37)$ & 0.837 \\
\hline \multicolumn{7}{|l|}{ Education } \\
\hline None & $125(18.9)$ & $535(81.1)$ & I & & 1 & \\
\hline Primary & $|7|$ (22.2) & $600(77.8)$ & $1.22(0.94,1.58)$ & 0.131 & $1.23(0.93,1.64)$ & 0.125 \\
\hline Secondary and above & $155(23.4)$ & $507(76.6)$ & I.3I (I, I.7I) & 0.046 & $1.25(0.92,1.70)$ & 0.129 \\
\hline \multicolumn{7}{|l|}{ Marital status } \\
\hline Single & $68(14.1)$ & $4 \mid 3(85.9)$ & 1 & & 1 & \\
\hline Married & $326(30.8)$ & $733(69.2)$ & $2.7(2.03,3.6)$ & $<0.001$ & $2.23(1.57,3.17)$ & $<0.001$ \\
\hline Separated & $57(10.3)$ & $496(89.7)$ & $0.7(0.48,1.02)$ & 0.06 & $0.58(0.37,0.91)$ & 0.020 \\
\hline \multicolumn{7}{|l|}{ Duration of sex work } \\
\hline Less than I year & $164(18.1)$ & $74 I(81.9)$ & I & & $\mathrm{I}$ & \\
\hline $\mathrm{I}-3$ years & $167(22.9)$ & $563(77.1)$ & $1.34(1.05,1.7 I)$ & 0.01 & $1.27(0.97,1.66)$ & 0.055 \\
\hline More than 3 years & $120(26.2)$ & $338(73.8)$ & $1.6(1.23,2.1)$ & $<0.001$ & $1.26(0.92,1.75)$ & 0.100 \\
\hline $\begin{array}{l}\text { Total number of sexual partners in } \\
\text { a week }\end{array}$ & & & & 0.760 & & \\
\hline One and less than one & $29(29.6)$ & $69(70.4)$ & I & & & \\
\hline More than one & $422(21.2)$ & $1573(78.8)$ & $0.64(0.4 I, I)$ & & & \\
\hline Number of working days in a week & & & & 0.683 & & \\
\hline Two and less than two & $42 I(2 I .2)$ & $1568(78.8)$ & 1 & & & \\
\hline More than two & $30(28.8)$ & $74(7 \mid .2)$ & $1.5 \mathrm{I}(0.97,2.34)$ & & & \\
\hline Consistent condom use with clients & & & & $<0.001$ & & $<0.001$ \\
\hline No & II $23(68.4)$ & $519(31.6)$ & I & & 1 & \\
\hline Yes & $360(79.8)$ & $91(20.2)$ & $1.83(1.42,2.35)$ & & $1.65(1.26,2.17)$ & \\
\hline Knowledge on ABC & & & & 0.018 & & 0.626 \\
\hline No & $257(23.6)$ & $833(76.4)$ & I & & 1 & \\
\hline Yes & $194(19.3)$ & $809(80.7)$ & $0.78(0.63,0.96)$ & & $1.04(0.82,1.37)$ & \\
\hline Knowledge on BCDEF & & & & 0.01 & & 0.210 \\
\hline No & 342 (22.9) & II $52(77.1)$ & I & & 1 & \\
\hline Yes & $109(18.2)$ & $490(81.8)$ & $0.75(0.59,0.95)$ & & $0.83(0.63,1.09)$ & \\
\hline Ever used drugs & & & & 0.020 & & 0.444 \\
\hline No & $43 I(22.1)$ & 1517 (77.9) & I & & 1 & \\
\hline Yes & $20(\mid 3.8)$ & $125(86.2)$ & $0.56(0.35,0.91)$ & & $0.8 \mathrm{I}(0.48,1.37)$ & \\
\hline Alcohol consumption & & & & 0.487 & & \\
\hline No & 140 (23.9) & $445(76.1)$ & I & & & \\
\hline Yes & $3 I I(20.6)$ & I I 97 (79.4) & $0.83(0.66,1.04)$ & & & \\
\hline Ever had an HIV test & & & & 0.031 & & 0.124 \\
\hline No & $186(19.2)$ & $784(80.8)$ & I & & 1 & \\
\hline Yes & $265(23.6)$ & $858(76.4)$ & $1.3(1.05,1.6 \mathrm{I})$ & & $0.80(0.60,1.06)$ & \\
\hline Met OEs/PEs/CMs in the last year & & & & 0.025 & & 0.026 \\
\hline No & $95(18)$ & $433(82)$ & I & & 1 & \\
\hline Yes & $356(22.7)$ & $1209(77.3)$ & $1.34(1.04,1.73)$ & & $1.42(1.04,1.93)$ & \\
\hline
\end{tabular}


Table 4 (Continued)

\begin{tabular}{|c|c|c|c|c|c|c|}
\hline \multirow[t]{2}{*}{ Variable } & \multicolumn{2}{|c|}{$\begin{array}{l}\text { Consistent condom use with } \\
\text { nonpaying partners }\end{array}$} & \multirow[t]{2}{*}{$\begin{array}{l}\text { Univariate OR } \\
(95 \% \mathrm{Cl})\end{array}$} & \multirow[t]{2}{*}{$P$-value } & \multirow[t]{2}{*}{$\begin{array}{l}\text { Multiple OR } \\
(95 \% \mathrm{CI})\end{array}$} & \multirow[t]{2}{*}{$P$-value } \\
\hline & $\begin{array}{l}\text { Yes }(N=45 \mathrm{I}, \\
21 \%), \mathrm{n}(\%)\end{array}$ & $\begin{array}{l}\text { No }(N=1642, \\
79 \%), \text { n (\%) }\end{array}$ & & & & \\
\hline Visited DIC in the last year & & & & $<0.001$ & & 0.356 \\
\hline No & $246(18.1)$ & $1110(81.9)$ & $\mathrm{I}$ & & I & \\
\hline Yes & $205(27.8)$ & $532(72.2)$ & $1.74(1.41,2.15)$ & & $1.140(0.86,1.5)$ & \\
\hline Visited STI clinic in the last year & & & & 0.653 & & \\
\hline No & $302(2 \mid .3)$ & II I8 (78.7) & $\mathrm{I}$ & & & \\
\hline Yes & $149(22.1)$ & $524(77.9)$ & $\mathrm{I} .05(0.84, \mathrm{I} .3 \mathrm{I})$ & & & \\
\hline Visited HTC center in the last year & & & & 0.754 & & \\
\hline No & $303(22.5)$ & $1046(77.5)$ & I & & & \\
\hline Yes & 148 (19.9) & $596(80.1)$ & $0.86(0.69,1.07)$ & & & \\
\hline
\end{tabular}

Note: Statistically significant values are shown in bold.

Abbreviations: ABC, A (abstinence from sex), B (monogamy or being faithful to one partner or avoiding multiple sex partners), and C (consistent and correct condom use or use of a condom during every sex act); BCDEF, B (monogamy or being faithful to one partner or avoiding multiple sex partners), $\mathrm{C}$ (consistent and correct condom use or use of a condom during every sex act), D (healthy-looking person can be infected with HIV), E (a person cannot get HIV from a mosquito bite), and $F$ (one cannot get HIV by sharing a meal with an HIV-infected person); Cl, confidence interval; CMs, community mobilizers; DIC, drop-in center; FSWs, female sex workers; HTC, HIV testing and counseling; OEs, outreach educators; OR, odds ratio; PEs, peer educators; STI, sexually transmitted infection.

multivariate analysis, year of survey, marital status and meeting with $\mathrm{OEs} / \mathrm{PEs} / \mathrm{CMs}$ in the last year and consistent condom use with regular clients were found to be associated with consistent condom use with nonpaying partners $(P<0.05)$. There was no statistically significant association of knowledge and misconceptions about HIV with consistent condom use with nonpaying partners. FSWs in 2015 had more than two times higher rate of condom use with nonpaying regular partners $(\mathrm{AOR}=2.89,95 \% \mathrm{CI}=2.08,4.01)$ than $\mathrm{FSWs}$ in 2006. Married FSWs were more likely to use condoms with regular nonpaying partners $(\mathrm{AOR}=2.23,95 \% \mathrm{CI}=1.57,3.17)$ than single FSWs. FSWs who met OEs/PEs/CMs in the last year were more likely to have condom use with nonpaying partners $(\mathrm{AOR}=1.42,95 \% \mathrm{CI}=1.04,1.93)$ than $\mathrm{FSWs}$ who did not meet $\mathrm{OEs} / \mathrm{PEs} / \mathrm{CMs}$ in the last year.

\section{Discussion}

Knowledge is the key factor to acquire awareness and predispose people to obtain good or bad things in their life. This study emphasized the HIV awareness and safe sexual behavior among FSWs in Kathmandu valley based on biobehavioral surveys from 2006 to 2015. It was found that the knowledge and misconception (BCDEF) on HIV/ AIDS was significantly decreased from 2008 to 2015 . These findings indicate a urgent need for programs on HIV/AIDS to prevent and control the consequences of disease in the country. ${ }^{12,13}$ Likewise, these findings encourage policy makers and program implementers to shift the focus of programs from raising general awareness of HIV/AIDS to educating FSWs about how HIV/AIDS is transmitted. Although improvements have been made in increasing knowledge and raising awareness, gaps of HIV knowledge still remain prevalent among FSWs. The deficiency of knowledge among FSWs as demonstrated in our study suggests the need for improvement in HIV training. Moreover, the significant drop in knowledge and misconceptions about HIV should be further investigated. Nonetheless, the rate of consistent condom use with clients and nonpaying partners significantly increased from 2008 to 2011. However, condom use with clients and nonpaying partners was not satisfactory in the 2014-2015 survey. Although use of condoms by FSWs with their regular clients was satisfactory (71\%), it was comparatively low (21\%) with nonpaying partners. This trend of unprotected sex with nonpaying partners poses a subsequently increased risk of HIV infection to FSWs and their partners. Findings of our study revealed that FSWs who have nonpaying partners have a large number of clients, which may act as the bridge of HIV/ AIDS infection to the general population. These findings also agreed well with others documented in the literature that concurrent partnerships with other FSWs, and low rates of condom use, led to higher prevalence of HIV among FSWs as compared to those FSWs with new and regular clients. ${ }^{20}$ Moreover, most of the FSWs receive economic support from regular nonpaying partners. ${ }^{20}$ Additionally, nonpaying partners frequently took FSWs as mistresses and provided them with steady economic support. ${ }^{21}$

Furthermore, it was found that HIV knowledge was not associated with safe sexual practices, while a shift in knowledge to condom use showed mixed results. A similar result was also reported in another study that the rate of condom use is low among FSWs even though knowledge on HIV and condoms is extensively promoted throughout the world. ${ }^{22}$ This unsafe sexual behavior acts as the main source of infection; therefore, to handle this issue, the HIV/AIDS awareness pro- 
gram acts as the key strategy for promoting knowledge and preventing and controlling the risk of HIV infection while making condom use consistent. ${ }^{23,24}$ For instance, it was found in many studies that knowledge on HIV prevention enables protective sexual behavior. ${ }^{25}$ Additionally, FSWs who had a sound knowledge on HIV/AIDS tended to reduce their numbers of partners and also use condoms during sexual intercourse, which resulted in them feeling protected against HIV, thereby increasing the rate of condom use as the partners became more familiar and intimate. In contrast, numerous studies show little impact on condom use with increased knowledge of its benefits. ${ }^{26,27}$

Education is strongly associated with knowledge and misconceptions about HIV/AIDs. This result is consistent with the results of other HIV/AIDS studies which have found an association between education and HIV/AIDS..$^{18,26}$ Peer-led HIV interventions, change of risky sexual behaviors, encouraging healthy sexual behaviors and modifying the norms are more effective in helping the members of a specific at-risk group for HIV/AIDS. ${ }^{28}$ Hence, this study indicated that HIV/AIDSrelated knowledge and safe sex behavior are influenced by exposure to HIV/AIDS programs. In fact, meeting with OEs/PEs/ CMs was strongly associated with safe sexual practices among FSWs. It was found that compared to professional health care providers, meeting with PEs was associated with less cost and was effective. ${ }^{28,29}$ A systematic review and meta-analysis of peer education interventions revealed that such interventions were significantly associated with increased HIV knowledge, reduced equipment sharing among injection drug users and increased rate of condom use. In addition, peer-based interventions have become a common method to effect important health-related behavior changes and are one of the most widely used strategies to address the HIV/AIDS pandemic. ${ }^{30,31}$ The findings of this study clearly point to the need of an HIV/AIDS program to promote HIV/AIDS knowledge and condom use.

The limitation of the study is the use of cross-sectional data that preclude analysis of the causal association between the determinants and outcome. This study also lacks data on potentially significant psychological factors (e.g., anxiety, depression) that may have influenced FSWs' self-reported behaviors. Moreover, this study took into account only the issues of violence and risk behaviors, while other issues such as legal policies and regulation of violence against FSWs have not been analyzed in detail.

\section{Conclusion}

Knowledge and misconceptions about HIV have decreased over years, suggesting a public health concern. Further study is needed to identify factors for the decreasing trend of knowledge and misconceptions about HIV. The rate of consistent condom use with clients and nonpaying partners has significantly increased. It was found that exposure to peer education was associated with safe sexual practices among FSWs and needs to be strengthened. There is urgent need of HIV intervention programs to increase knowledge and misconceptions about HIV among FSWs.

\section{Acknowledgments}

The authors are grateful to the participants, Integrated Biological and Behavioral Surveillance survey data collection research agencies, and staff who supported directly or indirectly in carrying out the surveys. They acknowledge the National Centre for AIDS and STD Control (NCASC), Teku, Kathmandu, Nepal, for their approval to use data.

\section{Disclosure}

The authors report no conflicts of interest in this work.

\section{References}

1. Baral S, Beyrer C, Muessig K, et al. Burden of HIV among female sex workers in low-income and middle-income countries: a systematic review and meta-analysis. Lancet Infect Dis. 2012;12(7):538-549.

2. Paz-Bailey G, Noble M, Salo K, Tregear SJ. Prevalence of HIV among US female sex workers: systematic review and meta-analysis. AIDS Behav. 2016;20(10):2318-2331.

3. Beyrer C, Crago AL, Bekker LG, et al. An action agenda for HIV and sex workers. Lancet. 2015;385(9964):287-301.

4. World Bank. HIV/AIDS in Nepal. 2012. Available from: http://www. worldbank.org/en/news/feature/2012/07/10/hiv-aids-nepal. Accessed November 21, 2017.

5. Paudel T, Singh N, Banjara MR, et al. Epidemiology of HIV, programmatic progress and gaps in last 10 years in Nepal. J Virus Erad. 2016;2(Suppl 4):35.

6. National Centre for AIDS and STD Control. Programmatic mapping and size estimation of key populations in South Africa: sex workers (male and female), men who have sex with men, persons who inject drugs and transgender people. Available from: http://www.aidsinfoonline.org/ kpatlas/document/ZAF/ZAF_2015_PSE_SW_MSM_PWID_TG.pdf. Accessed November 29, 2017.

7. National Center for AIDS and STD Control. Integrated Biological and Behavioral Surveillance (IBBS) survey among female sex workers in Pokhara valley, round V - 2016. Available from: https://www. aidsdatahub.org/sites/default/files/publication/Nepal_2016_Factsheet_ IBBS_survey_FSW_Pokhara_valley.pdf. Accessed December 19, 2017.

8. Kaufman MR, Harman JJ, Menger LM, et al. Understanding the experiences and needs of female commercial sex workers in Kathmandu, Nepal. Health Care Women Int. 2016;37(8):872-888.

9. Deuba, K, Anderson S, Ekström A, et al. Micro-level social and structural factors act synergistically to increase HIV risk among Nepalese female sex workers. Int J Infect Dis. 2016;49:100-106.

10. United Nations Education Scientific and Cultural Organization. Gender, Jobs and Education Prospects and Realities in Nepal. Kathmandu: UNESCO; 2014.

11. Paudel S. Women's concerns within Nepal's patriarchal justice system. Ethics in Action. 2011;5(6):30-36.

12. National Centre for AIDS and STD Control. Country Progress Report on HIV/AIDS Response, Nepal, 2014. Available from: http://www.unaids. org/sites/default/files/country/documents/NPL_narrative_report_2014. pdf. Accessed December 9, 2017. 
13. National Centre for AIDS and STD Control. Country Progress Report, Nepal, 2015. Available from: http://www.unaids.org/sites/default/ files/country/documents/NPL_narrative_report_2015.pdf. Accessed December 19, 2017.

14. Kakchapati S, Singh DR, Rawal BB, et al. Sexual risk behaviours, HIV and syphilis among female sex workers in Nepal. HIV AIDS (Auckl). 2017;9:9-18.

15. Shannon K, Strathdee SA, Goldenberg, et al. Global epidemiology of HIV among female sex workers: influence of structural determinants. Lancet. 2015;385(9962):55-71.

16. Ma MA, Phuengsamran D. Association between HIV/AIDs related knowledge and HIV/AIDS related behavior among urban young adult men in Indonesia. In: Proceedings of the 4th Asian Academic Society International Conference, Bangkok, Thailand, May 2016:301-307. Available from: http://aasic.org/proc/aasic/article/view/188.

17. Taffa N. Sexual activity of out-of-school youth, and their knowledge and attitude about STDs and HIV/AIDS in Southern Ethiopia. Ethiop J Health Dev. 2017;12(1):17-22.

18. Kakchapati S, Paudel T, Maharjan M, et al. Systematic differences in HIV, syphilis and risk behaviors among street based and establishment based female sex workers in Kathmandu valley of Nepal. Nepal J Epidemiol. 2016;6(4);620-630.

19. National Center for AIDS and STD Control. Integrated Biological and Behavioral Surveillance (IBBS) survey among people who inject drugs (PWID) in Kathmandu Valley, 2015, Round VI. Available from: http://www.aidsdatahub.org/sites/default/files/publication/ Nepal_IBBS_Full_Report_PWID_KTM_Rd6_2015.pdf. Accessed November 29, 2017.

20. Lowndes CM, Alary M, Gnintoungbe CA, et al. Management of sexually transmitted diseases and HIV prevention in men at high risk: targeting clients and non-paying sexual partners of female sex workers in Benin. AIDS. 2000;14:2523-2534.
21. Wolffers I, Triyoga RS, Basuki E, Yudhi D, Deville W, Hargono R. Pacar and Tamu: Indonesian women sex workers' relationships with men. Cult Health Sex. 1999;1:39-53.

22. Wong ML, Lubek I, Dy BC, et al. Social and behavioural factors associated with condom use among direct sex workers in SiemReap, Cambodia. Sex Transm Infect. 2003;79:163-165.

23. Weller S, Davis K. Condom effectiveness in reducing heterosexual HIV transmission. Cochrane Database Syst Rev. 2002;(1):CD003255.

24. Pinkerton SD, Abramson PR. Effectiveness of condoms in preventing HIV transmission. Soc Sci Med. 1997;44(9):1303-1312.

25. Magnani RJ, Karim AM, Weiss LA, et al. Reproductive health risk and protective factors among youth in Lusaka, Zambia. J Adolesc Health. 2002;30(1):76-86.

26. Essien EJ, Mgbere O, Monjok E, et al. Predictors of frequency of condom use and attitudes among sexually active female military personnel in Nigeria. HIV AIDS (Auckl). 2010;2:77-88.

27. Williams BG, Taljaard D, Campbell CM, et al. Changing patterns of knowledge, reported behaviour and sexually transmitted infections in a South African gold mining community. AIDS. 2003;17(14):2099-2107.

28. Webel AR, Okonsky J, Trompeta J, Holzemer WL. A systematic review of the effectiveness of peer-based interventions on health-related behaviors in adults. Am J Public Health. 2010;100:247-253.

29. Simoni JM, Nelson KM, Franks JC, Yard SS, Lehavot K. Are peer interventions for HIV efficacious? A systematic review. AIDS Behav. 2011;15:1589-1595.

30. Medley A, Kennedy C, O'Reilly K, Sweat M. Effectiveness of peer education interventions for HIV prevention in developing countries: a systematic review and meta-analysis. AIDS Educ Prev. 2009;21(3):181-206.

31. Tolli MV. Effectiveness of peer education interventions for HIV prevention, adolescent pregnancy prevention and sexual health promotion for young people: a systematic review of European studies. Health Educ Res. 2012;27(5):904-913.
HIV/AIDS - Research and Palliative Care

\section{Publish your work in this journal}

HIV/AIDS - Research and Palliative Care is an international, peerreviewed open access journal focusing on advances in research in HIV, its clinical progression and management options including antiviral treatment, palliative care and public healthcare policies to control viral spread. The journal is included in PubMed. The manuscript man-

\section{Dovepress}

agement system is completely online and includes a very quick and fair peer-review system, which is all easy to use. Visit http://www.dovepress. com/testimonials.php to read real quotes from published authors. 